Canadian

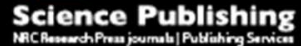

Canadian Journal of Physiology and Pharmacology Revue canadienne de physiologie et pharmacologie

\title{
Addition of Dexmedetomidine to QX-314 Enhances the Onset and Duration of Sciatic Nerve Block in Rats
}

\begin{tabular}{|c|c|}
\hline Journal: & Canadian Journal of Physiology and Pharmacology \\
\hline Manuscript ID & cjpp-2017-0331.R2 \\
\hline Manuscript Type: & Article \\
\hline Date Submitted by the Author: & 28-Jul-2017 \\
\hline Complete List of Authors: & $\begin{array}{l}\text { Zhao, Wenling; Laboratory of Anesthesia and Critical Care Medicine \& } \\
\text { Translational Neuroscience Centre, West China Hospital, Sichuan } \\
\text { University, Chengdu } 610041 \text {, Sichuan, China } \\
\text { Yin, Qinqin; Laboratory of Anesthesia and Critical Care Medicine \& } \\
\text { Translational Neuroscience Centre, West China Hospital, Sichuan } \\
\text { University, Chengdu } 610041 \text {, Sichuan, China } \\
\text { Liu, Jin; Laboratory of Anesthesia and Critical Care Medicine, Translational } \\
\text { Neuroscience Center, West China Hospital, Sichuan University, Chengdu } \\
610041 \text {, Sichuan, P. R. China } \\
\text { Zhang, Wensheng; Laboratory of Anesthesia and Critical Care Medicine, } \\
\text { Translational Neuroscience Center, West China Hospital, Sichuan } \\
\text { University, Chengdu 610041, Sichuan, P. R. China } \\
\text { Yang, Linghui; Laboratory of Anesthesia and Critical Care Medicine \& } \\
\text { Translational Neuroscience Centre, West China Hospital, Sichuan } \\
\text { University, Chengdu } 610041 \text {, Sichuan, China }\end{array}$ \\
\hline $\begin{array}{l}\text { Is the invited manuscript for } \\
\text { consideration in a Special } \\
\text { Issue?: }\end{array}$ & N/A \\
\hline Keyword: & dexmedetomidine, QX-314, nerve block, drug combination \\
\hline
\end{tabular}




\section{Addition of Dexmedetomidine to QX-314 Enhances the Onset and Duration of Sciatic Nerve Block in Rats}

Wenling Zhao, Qinqin Yin, Jin Liu, Wensheng Zhang, and Linghui Yang

W. L. Zhao, Q. Q. Yin , J. Liu, W. S. Zhang, and L. H. Yang. Laboratory of Anesthesia and Critical Care Medicine \& Translational Neuroscience Centre, West China Hospital, Sichuan University, Chengdu 610041, Sichuan, China

Corresponding author: Wensheng Zhang (Tel: +86-2885164040; E-mail: zhang_ws@scu.edu.cn)

Conflicts of interest: The authors have no conflicts of interest.

Funding: Supported by the grant 2014ZX09101001-003 (to Dr. Zhang) from the National Science and Technology Major Project of China, Beijing, P.R. China. 


\section{Abstract}

An experimental set-up was designed to observe whether adding dexmedetomidine to QX-314 would enhance the onset and duration of sensory and motor function in a rat sciatic nerve block model. Fifty-six Sprague-Dawley rats received unilateral sciatic nerve blocks with $0.2 \mathrm{ml}$ of $35 \mathrm{mM}$ QX-314 alone, dexmedetomidine (5.3 $\mu \mathrm{M}[1$ $\left.\left.\mu \mathrm{g} \cdot \mathrm{kg}^{-1}\right], 26.4 \mu \mathrm{M}\left[5 \mu \mathrm{g} \cdot \mathrm{kg}^{-1}\right], 52.8 \mu \mathrm{M}\left[10 \mu \mathrm{g} \cdot \mathrm{kg}^{-1}\right]\right)$ alone, or a combination of the two. Thermal nociception and motor function were assessed by a blinded investigator, sciatic nerves and perineural tissues were harvested at 14 days after injection. In addition, we examined the effects of these solutions on compound action potentials in isolated frog sciatic nerves. Dexmedetomidine added to QX-314 enhanced the onset and duration of thermal nociception block and motor block $(P<0.05)$ without aggravating histopathological injuries. Furthermore, $52.8 \mu \mathrm{M}$ dexmedetomidine added to $35 \mathrm{mM}$ QX-314 showed less inflammation than QX-314 alone at 14 days $(P$ $=0.003)$. Dexmedetomidine plus QX-314 was shown to dose-dependently reduce the compound action potentials relative to QX-314 alone $(P<0.05)$. It was concluded that coadministration of QX-314 with a clinical-dose of dexmedetomidine produced a synergistic anesthetic effect to enhance the effect of sciatic nerve block.

Keywords: dexmedetomidine, QX-314, nerve block, drug combination 


\section{Introduction}

The conventional long-acting local anesthetics such as ropivacaine, produce shortterm analgesia for postoperative pain relief, particularly in the early stage. There are only a few drugs that can provide long-lasting analgesia. For example, Exparel (Pacira Pharmaceuticals, Inc., California, USA), a liposomal bupivacaine, can produce postoperative analgesia for up to three days as reported. However, intravenous opioid drugs are needed in conjunction, bringing about many side effects. Furthermore, it is generally known that QX-314, a quaternary lidocaine derivative, provides long-lasting anesthesia with slow onset and low efficacy owing to its positive charge (Kosugi et al. 2010; Lim et al. 2007). It has been shown that QX-314 can be administered together with capsaicin or acid solution to produce a nociceptor selective, long-lasting rat sciatic nerve block (Binshtok et al. 2007; Liu et al. 2011). However, local injection of these additives might cause severe neurotoxicity. There is therefore an increased interest to find new or adjuvant drugs that will provide longlasting analgesia with minimal side effects (Caterina et al. 1997; Rukwied et al. 2007).

Dexmedetomidine, a potent $\alpha_{2}$-adrenoceptor agonist, has been shown to increase the analgesic effects of local anesthetics (Esmaoglu et al. 2010; Gupta et al. 2011). Animal studies demonstrated that dexmedetomidine prolongs the duration of sensory and motor blocks when added to local anesthetics without aggravating the nerve and muscle injuries (Ali Erdogan et al. 2013; Brummett et al. 2008; Brummett et al. 2009). The efficacy of dexmedetomidine plus local anesthetics for peripheral nerve blocks or intrathecal anesthesia in human studies has also been established (Safari et al. 2016). We previously observed that a combination of dexmedetomidine and QX314 enhanced the duration of nerve blockade in rats (data not presented). 
Taken together, we hypothesized that adding dexmedetomidine to QX-314 would enhance the effectiveness of QX-314-mediated sciatic nerve block while not aggravating the tissue toxicity. Further, we were also interested in investigating whether dexmedetomidine alone or added to QX-314 can affect action potential conduction in vitro. Hence we recorded compound action potentials (CAPs) from isolated frog sciatic nerves.

\section{Methods}

\section{Animals}

The study was approved by the Institutional Animal Experimental Ethics Committee of Sichuan University with the approval number: 2015014A. All the animals were purchased from the Experimental Animal Center of Sichuan province, adult male Sprague-Dawley (SD) rats and frogs (Rana catesbianaofeither sex). The handling and experimental procedures were consistent with the Guide for the Care and Use of Laboratory Animals (Vol. 1, 2nd ed., 1993, from the Canadian Council on Animal Care).

\section{Drug preparation}

Commercially available dexmedetomidine hydrochloride (Jiangsu Hengrui Medicine Co. Ltd., Jiangsu, China) was made up to the concentrations of $5.3 \mu \mathrm{M}$, $26.4 \mu \mathrm{M}, 52.8 \mu \mathrm{M}$ with $0.9 \%$ normal saline, and with or without $35 \mathrm{mM}$ QX-314 (Sigma-Aldrich Co. Ltd., Shanghai, China). This concentration of QX-314 was based on a preliminary study (Supplemental material, Table 1), $35 \mathrm{mM}$ was the minimal effective concentration to block sciatic nerve completely (data not shown). According 
to the average weight $(250 \mathrm{~g})$ of rats, the doses of dexmedetomidine were approximately equal to $1 \mu \mathrm{g} \cdot \mathrm{kg}-1,5 \mu \mathrm{g} \cdot \mathrm{kg}-1$ and $10 \mu \mathrm{g} \cdot \mathrm{kg}-1$ and were the clinically common doses (Brummett et al. 2009). The $\mathrm{pH}$ of these solutions, ranging between 6.2 and 7.2, was assumed to buffer quickly around the tissue.

\section{Experiment 1: Sciatic nerve block in rats}

\section{Groups}

The rats were housed on a 7:00 AM to 7:00 PM light-dark cycle and had unlimited access to food and water. All rats used in the experiment were used to handling. They had a minimum of seven days to adapt to the neurobehavioral facility before commencing the experiment. Fifty-six rats $\left(n=8 \cdot \operatorname{group}^{-1}\right)$ were divided into seven groups and treated with QX-314 $(35 \mathrm{mM})$ alone or dexmedetomidine $(5.3 \mu \mathrm{M}, 26.4$ $\mu \mathrm{M}, 52.8 \mu \mathrm{M})$ with or without QX-314 (35 mM). At the time of administration, the rats weighed approximately 240 to $262 \mathrm{~g}$. The experimental design was that of a double-blinded procedure.

\section{Sciatic nerve block model}

Rats were briefly anesthetized by inhalation $2-3 \%$ isoflurane in oxygen, and the midpoint of landmarks(greater trochanter and ischial tuberosity) in the left hind limb were localized. A 26-gauge injection syringe was introduced to the ischiatic notch, then $0.2 \mathrm{~mL}$ of the test drug was injected once in contact with the bone (Gerner et al., 2008; Thalhammer et al. 1995). The right limb was always kept as a blank control to which no drugs were administered.

\section{Neurobehavioral assessment}


The assessment was performed at the following intervals after injection: $15 \mathrm{~min}, 30$ min, 1 h, 2 h, 3 h, 4 h, 6 h, 8 h, 10 h until both the sensory and motor values were up to the baselines.

Thermal nociception was investigated by using a modified hot plate test (Shankarappa et al. 2012). The fixed anatomic area of hind paws were exposed to a $56 \square$ hot plate (model RB-200 hotplate analgesia metre; Chengdu Technology \& market Cos. Ltd., Chengdu, China) in sequence (left then right). The time of latency until paw withdrawal (PWL) was recorded by a stopwatch. The investigator could remove the paw to avoid empyrosis or hyperalgesia if the PWL $>12 \mathrm{~s}$ (the cut-off value). PWL $>6 \mathrm{~s}$ were considered successful nerve block. The onset time of thermal nociception block was investigated as the PWL $>6 \mathrm{~s}$ form baseline. The duration of effective nociception blockade was calculated as the time greater than or equal to a value of $6 \mathrm{~s}$.

Motor function was estimated by measuring the postural extensor thrust (PET) of the left hind limb (Shankarappa et al. 2012). The rats were suspended over a balance and the maximum force that resisted contact of a digital platform (model HZT-B5000; Huazhi scientific instrument Co. Ltd., Fujian, China) by the heel was measured. The following score standards were used: 0 , no block, PET = baseline; 1 , minimal block, $50 \% \leq$ PET $<100 \%$ of baseline; 2 , moderate block, $20 \mathrm{~g} \leq$ PET $<50 \%$ of baseline; 3 , complete block, PET $<20$ g. A score higher than 2 was considered a successful nerve block. The duration of effective motor function blockade was calculated as the time greater than or equal to score 2.

The neurobehavioral evaluation was repeated three times at each point and calculated as the mean value to increase accuracy. 


\section{Experiment 2: Tissue harvesting and histology}

To evaluate the pathology of the tissue, rats from Experiment 1 were euthanatized with $2 \mathrm{ml}$ propofol at 14 days after drug injection. The sciatic nerves and adjacent tissues (2 cm long at the site of injection) were harvested and stained with haematoxylin-eosin following standard techniques (Padera et al. 2008). Briefly, samples were fixed in $10 \%$ neutral formalin for one day and transferred to an ethanol solution for dehydration. They were subsequently embedded in paraffin, cut by length $(4 \mu \mathrm{m})$ and stained with haematoxyin and eosin. The histopathological examinations were analyzed by a pathologist, blinded to drug treatment. The scoring standard has previously been described for inflammation (0-4) and myotoxicity (0-6) (Padera et al. 2006). The former is decided by the degree of inflammatory corpuscle. The scores assessing the degree of muscle injury are: 0 , normal tissue; 1, perifascicular internalisation; 2, deep internalisation (over five cell layers); 3,perifascicular regeneration; 4, deep regeneration; 5, hemifascicular regeneration; and 6, holofascicular regeneration.

\section{Experiment 3:Recording the CAPs from isolated frog sciatic nerves}

Frogs (40-65 g) were pithed and dissected. Thirty-six sciatic nerves $(4-5 \mathrm{~cm}$ long) were obtained from the lumbar plexus to the knee and then put into a Ringer's solution. Ringer's solution was made dissolving $6.50 \pm 0.02 \mathrm{~g} \mathrm{NaCl}, 0.14 \pm 0.01 \mathrm{~g}$ $\mathrm{KCl}, 0.12 \pm 0.01 \mathrm{~g} \mathrm{CaCl}_{2}, 0.20 \pm 0.01 \mathrm{~g} \mathrm{NaHCO}_{3}$ and $0.01 \mathrm{~g} \mathrm{NaH}_{2} \mathrm{PO}_{4}$ in one litre of distilled water $(\mathrm{pH} 7.0 \pm 0.1)$. This solution was stirred at $300 \mathrm{rpm}$ with a magnetic stirrer (MS-H280-Pro; SCILOGEX LLC, Berlin, CT 06037 USA) to keep the homogeneity of the Ringer's solution around the isolated sciatic nerves. They were subsequently randomly divided into six groups for further treatment: $35 \mathrm{mM} \mathrm{QX-314}$ 
alone, $26.4 \mu \mathrm{M}$ and $52.8 \mu \mathrm{M}$ dexmedetomidine alone, or $5.3 \mu \mathrm{M}, 26.4 \mu \mathrm{M}$ and 52.8 $\mu \mathrm{M}$ dexmedetomidine with $35 \mathrm{mM}$ QX-314. All the drugs dissolved in Ringer's solution instead of $0.9 \%$ normal saline. The $\mathrm{pH}$ of the test solutions was adjusted to $7.0 \pm 0.1$ with $\mathrm{HCl}$ or $\mathrm{NaOH}$ (Katsuki et al. 2006).

Before the start of the experiment, when the frog sciatic nerves were soaked in the Ringer's solution for 20 min (Kosugi et al. 2010), the CAPs were recorded every five minutes to set the baseline values. Action potentials from the isolated frog sciatic nerves were recorded by the BL-420F biological signal acquisition and analysis system (Techman Software Co. LTD, Chengdu, China), using the air-gap method ${ }^{2}$. The parameter settings were: frequency, $100 \mathrm{~Hz}$; duration of rectangular pulses, 0.1 ms; voltage, $1 \mathrm{~V}$. Measurements were recorded quickly (15 s at the most) after being soaked into the test solutions for the following intervals: $5 \mathrm{~min}, 10 \mathrm{~min}, 15 \mathrm{~min}, 20$ min, $30 \mathrm{~min}, 45 \mathrm{~min}$ and $60 \mathrm{~min}$. Each frog sciatic nerve fiber was only used once to evaluate the effect of a test drug on CAPs. All the measurements were collected at room temperature (24-27 $\square$, regulated by an air-conditioner).

\section{Statistical Analysis}

The data were analyzed using SPSS 22.0 (IBM SPSS Inc., Chicago, USA). The neurobehavioral data and histopathologic scores are presented as medians with interquartile ranges because they are not normally distributed (the assumption of a normal distribution was confirmed using the Shapiro-Wilk test). To assess the statistical significance of neurobehavioral examinations between QX-314 and QX314 plus $5.3 \mu \mathrm{M}$ dexmedetomidine at each time point, nonparametric analysis was used. The Mann-Whitney U test with Bonferroni's correction $(\alpha=0.05 / 6)$ was used for multiple comparisons of duration of effective block, and Bonferroni's correction ( $\alpha$ 
$=0.05 / 10)$ was completed for multiple comparisons of histopathologic examinations.

For Experiment 3, data of CAPs are shown as mean \pm standard error of mean (SEM).

A repeated-measures ANOVA evaluated the effects of relative CAP amplitude between groups. The statistical significance was established at $P<0.05$.

\section{Results}

\section{Experiment 1: The effect of sciatic nerve block in rats}

Rats were tested for both thermal nociception and motor function blockades by using the hotplate and postural extensor test, respectively. The onset time of thermal nociception block was approximately $2 \mathrm{~h}$ for $35 \mathrm{mM}$ QX-314, but $30 \mathrm{~min}$ when QX314 was administered together with three doses of dexmedetomidine (Fig. 1A). Addition of dexmedetomidine to $35 \mathrm{mM}$ QX-314 showed similar effects on motor function block (Fig. 1B). Thermal nociception and motor function measured at individual time points from $15 \mathrm{~min}$ to $10 \mathrm{~h}$ after injection showed significant differences when comparing 5.3 $\mu \mathrm{M}$ dexmedetomidine added to $35 \mathrm{mM}$ QX-314 and 35 mM QX-314 alone (Fig. 1, C-D, $P<0.05$ ). But the effects of sciatic nerve block (both sensory and motor function) were not statistically significant at anytime point between the three concentrations of dexmedetomidine/QX-314 $(P>0.05)$.

Duration of effective thermal nociception and motor function blockades were prolonged when $35 \mathrm{mM}$ QX-314 was coadministered with dexmedetomidine $(5.3 \mu \mathrm{M}$, $26.4 \mu \mathrm{M}, 52.8 \mu \mathrm{M}$ ), compared with QX-314 alone (Fig. 2, $P<0.0083$ ). Similarly, there were no statistically significant differences between the three doses of dexmedetomidine/QX-314 $(P>0.0083)$.

In addition, concentrations of $5.3 \mu \mathrm{M}, 26.4 \mu \mathrm{M}$ and $52.8 \mu \mathrm{M}$ dexmedetomidine 
exhibited no effective sciatic nerve block (Fig. 3, A, C, E). The right limbs (negative controls, without drug administered) of the three groups of QX-314 plus dexmedetomidine did not produce analgesia or motor block (Fig. 3, B, D, F). For all the rats, the time to resumption of righting reflex was less than $15 \mathrm{~min}$. Therefore, there was little systemic analgesic effect of dexmedetomidine.

\section{Experiment 2: Tissue Harvesting and Histology}

In all cases, tissues had a benign appearance, with mild or moderate inflammation at 14 days. Rats injected with one of the three concentrations of dexmedetomidine alone showed no or little inflammation. Compared with the positive control (samples of 23 $\mathrm{mM}$ bupivacaine; obtained from preliminary study), microscopic examination of the tissues injected with $35 \mathrm{mM}$ QX-314 revealed similar inflammatory reaction $(P>$ 0.005). Dexmedetomidine $(52.8 \mu \mathrm{M})$ administered alongside $35 \mathrm{mM}$ QX-314 alleviated the inflammation relative to $35 \mathrm{mM}$ QX-314 alone (Fig. 4A, $P=0.003<$ 0.005). All sections showed no apparent injury of muscle (Fig. 4B).

\section{Experiment 3: The CAPs of isolated frog sciatic nerve}

As shown in the Figure 5A, soaking the frog sciatic nerve fibers in Ringer's solution containing dexmedetomidine $(26.4 \mu \mathrm{M}$ or $52.8 \mu \mathrm{M})$ did not change the peak amplitudes of CAPs, and the relative CAPs amplitudes showed no significant differences between the two solutions during soaking for $60 \mathrm{~min}$.

Adding dexmedetomidine $(5.3 \mu \mathrm{M}, 26.4 \mu \mathrm{M}$ or $52.8 \mu \mathrm{M})$ to $35 \mathrm{mM}$ QX-314 inhibited the peak amplitudes of CAPs (Fig. 5, B-C). There were statistically significant differences between all four treatment groups $(P<0.05)$. 


\section{Discussions}

In this study, we demonstrated that $35 \mathrm{mM}$ QX-314 alone produced short-acting effective thermal nociception block. We furthermore demonstrated that the addition of dexmedetomidine to QX-314 greatly enhanced the effect on nerve blockade of both thermal nociception and motor function in a rat sciatic nerve blockade model, and attenuated the inflammatory response when $52.8 \mu \mathrm{M}$ dexmedetomidine was added. Dexmedetomidine is therefore thought to be a good additive to QX-314 to produce long-lasting local anesthesia in the future. Our data also showed that the administration of dexmedetomidine in combination with QX-314 inhibited the CAPs of frog sciatic nerve fibers dose-dependently in vitro. This suggests that dexmedetomidine can affect the conduction of action potentials at the local nerve level.

Dexmedetomidine was first proposed as an adjuvant to lidocaine for intravenous regional anesthesia to improve the quality of perioperative analgesia without causing sideeffects by Memis (Memis et al. 2004). In addition, a combination of highconcentration dexmedetomidine and bupivacaine has been shown to significantly increase the duration of bupivacaine-induced antinociception in rat sciatic nerve blockade without neurotoxicity (Brummett et al. 2009). Indeed, previous research and clinical studies have demonstrated the efficacy when dexmedetomidine was added to frequently-used local anesthetics. For example, a single-centre, prospective, randomized, three-blind and controlled trial (Fritsch et al. 2014), showed that the addition of dexmedetomidine to ropivacaine for interscalene blocks increased the duration of nerve block and improved postoperative pain without side effects or neurological complication (reference needed). Mahendru (Mahendru et al. 2013) concluded that intrathecal dexmedetomidine can prolong both the motor and sensory 
block as well as maintain the hemodynamic stability when compared to clonidine.

QX-314, a quaternary lidocaine derivative, has recently gained interest because of its potential application in prolonged or sensory-selective regional anesthesia (Binshtok et al. 2007). In our study, 35 mM QX-314 only produced about two hours of effective thermal nociception block with mild inflammation and muscle injury, as reported previously (Shankarappa et al. 2012). Shankarappa demonstrated that longacting anesthesia is achieved only with a high concentration of QX-314 $(70 \mathrm{mM})$, resulting in associated severe tissue toxicity making it thereby inappropriate for clinical use. Since then efforts have been made to minimize its neurotoxicity and capitalize on the long-lasting anesthetic properties of QX-314. Some researchers (Binshtok et al. 2007) reported how QX-314 can block sodium channels in the existence of capsaicin, a transient receptor potential cation channel subfamily $\mathrm{V}$ member 1 (TRPV1) agonist. Binshtok (Binshtok et al. 2009) used lidocaine instead of capsaicin to delivery QX-314 into nociceptors through TRPV1 channels. Sagie and Kohane (Sagie and Kohane 2010) demonstrated that certain doses of surfactants (sodium octyl sulfate, octyltrimethylammonium bromide, Tween 20) coadministered with QX-314 can produce long-acting and sensory-selective nerve block. Here we found that dexmedetomidine may have a similar effect.

There are many probable mechanisms of action for dexmedetomidine improving QX-314-mediated nerve block, such as a direct action in the peripheral nerve, centrally-mediated analgesia, vascular constriction by $\alpha 2$-adrenoceptor, or as a TRP channel opener. In the present study, rats displayed unilateral blocks with a blank control paw, standing for central analgesia, so the effects were predominately at the peripheral nerve level. Also, the isolated frog sciatic nerve experiment showed that low concentrations of dexmedetomidine $(26.4 \mu \mathrm{M}$ and $52.8 \mu \mathrm{M})$ did not affect the 
CAPs. This is in agreement with Kosugi (Kosugi et al. 2010), showing that only high-concentration dexmedetomidine reduced the peak amplitude of CAPs (IC50 = $400 \mu \mathrm{M})$. However, our data showed a dose-dependent effect on the inhibition of the peak amplitude of CAPs when administering dexmedetomidine with QX-314. This observation can evidence the effect of dexmedetomidine on peripheral nerve block. Brummett (Brummett et al. 2011) demonstrated that the analgesic effect of dexmedetomidine can be reversed by pretreating with an hyperpolarization-activated current enhancer, not an a2-adrenoceptor antagonist. Considering QX-314's characteristics, the prolongation of nerve block may reflect a dexmedetomidineinduced entry of QX-314 into the peripheral nerve through pathways such as TRP channels (Binshtok et al. 2007). Regional injection of capsaicin, acid solution, local anesthetics, emulsified isoflurane or heat exposure can deliver QX-314 into sensory neurons by activating TRPV1 channels to provide a rapid onset and long-acting nociceptor-selective block (Binshtok et al. 2007; Liu et al. 2011; Romanovsky et al. 2009; Zhou et al. 2014; Brenneis et al. 2014). However, so far no research has suggested that dexmedetomidine could open a TRP channel. Therefore, further experiments are needed to clarify the exact mechanism underlying perineural administration of dexmedetomidine in combination with QX-314 for prolonged local anesthesia.

We found that perinerual administration of $52.8 \mu \mathrm{M}$ dexmedetomidine with $35 \mathrm{mM}$ QX-314 alleviated the inflammation when compared to $35 \mathrm{mM}$ QX-314 alone. Similarly, the inflammatory reaction of bupivacaine decreased when coadministered with 120.6 $\mu \mathrm{M}$ dexmedetomidine (Brummett et al. 2009). Further, recent research (Huang et al. 2014) demonstrated that $120.6 \mu \mathrm{M}$ dexmedetomidine can have an antiinflammatory effect by reducing inflammatory cytokines through inhibiting the 
translocation of activated nuclear factor- $\mathrm{B}$ to the nucleus.

There were some limitations to this study. First, the sensory measure in our experiment was limited to the reaction to a heat stimulus. Other types of nociception like mechanical stimulation could be tested. Second, although the isolated frog sciatic nerve study could show the direct dose-dependent effect of dexmedetomidine plus QX-314 on peripheral nerve block, this is an in vitro study. It therefore cannot mimic a two-drug mechanisms of action in vivo, for a large fraction of drugs will be metabolized or not even reach the nerve fibres in rodent studies. Further studies will be required to fully elucidate the exact mechanism of action of dexmedetomidine.

In summary, this animal study verified the hypothesis that dexmedetomidine can enhance the onset and duration of QX-314-induced anesthesia in rat sciatic nerve block, and inhibit the peak amplitudes of CAPs in frog sciatic nerves in vitro. Furthermore, adding $52.8 \mu \mathrm{M}$ dexmedetomidine to QX-314 can alleviate the perineural inflammatory response 14 days after drug administration. Accordingly, dexmedetomidine may be a good adjuvant to QX-314 for long-acting anesthesia.

\section{References}

Ali Erdogan, M., Polat, A., Yucel, A., Aydogan, M. S., Parlakpinar, H., Tekin, S., et al. 2013. Effects of perineural administration of dexmedetomidine in combination with levobupivacaine in a rat sciatic nerve block. Curr Ther Res Clin Exp. 74: 74-78. doi:10.1016/i.curtheres.2013.03.001. PMID:24385106. Binshtok, A. M., Bean, B. P., \& Woolf, C. J. 2007. Inhibition of nociceptors by TRPV1-mediated entry of impermeant sodium channel blockers. Nature. 449(7162): 607-610. doi:10.1038/nature06191. PMID:17914397.

Binshtok, A. M., Gerner, P., Oh, S. B., Puopolo, M., Suzuki, S., Roberson, D. P., et al. 2009. Coapplication of lidocaine and the permanently charged sodium channel blocker QX-314 produces a long-lasting nociceptive blockade in rodents. Anesthesiology, 111(1): 127-137. doi: 10.1097/ALN.0b013e3181a915e7. PMID:19512868.

Brenneis, C., Kistner, K., Puopolo, M., Jo, S., Roberson, D., Sisignano, M., et al. 2014. Bupivacaineinduced cellular entry of QX-314 and its contribution to differential nerve block. Br J Pharmacol. 171(2): 438-451. doi: 10.1111/bph.12466. PMID:24117225.

Brummett, C. M., Norat, M. A., Palmisano, J. M., \& Lydic, R. 2008. Perineural administration of 
dexmedetomidine in combination with bupivacaine enhances sensory and motor blockade in sciatic nerve block without inducing neurotoxicity in rat. Anesthesiology. 109(3): 502-511. doi: 10.1097/ALN.0b013e318182c26b. PMID:18719449.

Brummett, C. M., Padda, A. K., Amodeo, F. S., Welch, K. B., \& Lydic, R. 2009. Perineural dexmedetomidine added to ropivacaine causes a dose-dependent increase in the duration of thermal antinociception in sciatic nerve block in rat. Anesthesiology. 111(5): 1111-1119. doi: 10.1097/ALN.0b013e3181bbcc26. PMID:19858875.

Brummett, C. M., Hong, E. K., Janda, A. M., Amodeo, F. S., \& Lydic, R. 2011. Perineural dexmedetomidine added to ropivacaine for sciatic nerve block in rats prolongs the duration of analgesia by blocking the hyperpolarization-activated cation current. Anesthesiology. 115(4): 836-843. doi:10.1097/ALN.0b013e318221fcc9. PMID:21666435.

Caterina, M. J., Schumacher, M. A., Tominaga, M., Rosen, T. A., Levine, J. D., \& Julius, D. 1997. The capsaicin receptor: a heat-activated ion channel in the pain pathway. Nature. 389(6653): 816-824. doi: 10.1038/39807. PMID:9349813.

Esmaoglu, A., Yegenoglu, F., Akin, A., \& Turk, C. Y. 2010. Dexmedetomidine added to levobupivacaine prolongs axillary brachial plexus block. Anesth Analg. 111(6): 1548-1551. doi: 10.1213/ANE.0b013e3181fa3095. PMID:20889939.

Fritsch, G., Danninger, T., Allerberger, K., Tsodikov, A., Felder, T. K., Kapeller, M., et al. 2014. Dexmedetomidine added to ropivacaine extends the duration of interscalene brachial plexus blocks for elective shoulder surgery when compared with ropivacaine alone: a single-center, prospective, triple-blind, randomized controlled trial. Reg Anesth Pain Med. 39(1): 37-47. doi: 10.1097/AAP.0000000000000033. PMID:24317234.

Gerner, P., Binshtok, A. M., Wang, C. F., Hevelone, N. D., Bean, B. P., Woolf, C. J., et al. 2008. Capsaicin combined with local anesthetics preferentially prolongs sensory/nociceptive block in rat sciatic nerve. Anesthesiology. 109(5): 872-878. doi:10.1097/ALN.0b013e31818958f7. PMID:18946300.

Gupta, R., Bogra, J., Verma, R., Kohli, M., Kushwaha, J. K., \& Kumar, S. 2011. Dexmedetomidine as an intrathecal adjuvant for postoperative analgesia. Indian J Anaesth. 55(4): 347-351. doi: 10.4103/00195049.84841. PMID:22013249.

Huang, Y., Lu, Y., Zhang, L., Yan, J., Jiang, J., \& Jiang, H. 2014. Perineural dexmedetomidine attenuates inflammation in rat sciatic nerve via the NF-kappaB pathway. Int J Mol Sci. 15(3): 4049-4059. doi: 10.3390/ijms15034049. PMID:24663080.

Katsuki, R., Fujita, T., Koga, A., Liu, T., Nakatsuka, T., Nakashima, M., et al. 2006. Tramadol, but not its major metabolite (mono-O-demethyl tramadol) depresses compound action potentials in frog sciatic nerves. Br J Pharmacol. 149(3): 319-327. doi:10.1038/sj.bjp.0706868. PMID: 16921387.

Kosugi, T., Mizuta, K., Fujita, T., Nakashima, M., \& Kumamoto, E. 2010. High concentrations of dexmedetomidine inhibit compound action potentials in frog sciatic nerves without alpha(2) adrenoceptor activation. Br J Pharmacol. 160(7): 1662-1676. doi:10.1111/j.1476-5381.2010.00833.x. PMID: 20649570.

Lim, T. K., Macleod, B. A., Ries, C. R., \& Schwarz, S. K. 2007. The quaternary lidocaine derivative, QX314 , produces long-lasting local anesthesia in animal models in vivo. Anesthesiology. 107(2): 305-311. doi:10.1097/01.anes.0000270758.77314.b4. PMID:17667576.

Liu, H., Zhang, H. X., Hou, H. Y., Lu, X. F., Wei, J. Q., Wang, C. G., et al. 2011. Acid solution is a suitable medium for introducing QX-314 into nociceptors through TRPV1 channels to produce sensory-specific analgesic effects. PLoS One. 6(12): e29395. doi:10.1371/journal.pone.0029395. PMID:22216270.

Mahendru, V., Tewari, A., Katyal, S., Grewal, A., Singh, M. R., \& Katyal, R. 2013. A comparison of intrathecal dexmedetomidine, clonidine, and fentanyl as adjuvants to hyperbaric bupivacaine for lower limb surgery: A double blind controlled study. Journal of Anaesthesiology Clinical Pharmacology. 29(4): 496-502. doi:10.4103/0970-9185.119151. PMID:24249987.

Memis, D., Turan, A., Karamanlioglu, B., Pamukcu, Z., \& Kurt, I. 2004. Adding dexmedetomidine to lidocaine for intravenous regional anesthesia. Anesth Analg. 98(3): 835-840, table of contents. PMID: 14980948.

Padera, R. F., Tse, J. Y., Bellas, E., \& Kohane, D. S. 2006. Tetrodotoxin for prolonged local anesthesia with minimal myotoxicity. Muscle Nerve. 34(6): 747-753. doi:10.1002/mus.20618. PMID: 16897761.

Padera, R., Bellas, E., Tse, J. Y., Hao, D., \& Kohane, D. S. (2008). Local myotoxicity from sustained release of bupivacaine from microparticles. Anesthesiology. 108(5): 921-928. doi: 10.1097/ALN.0b013e31816c8a48. PMID:18431129. 
Romanovsky, A. A., Almeida, M. C., Garami, A., Steiner, A. A., Norman, M. H., Morrison, S. F., et al. 2009. The transient receptor potential vanilloid-1 channel in thermoregulation: a thermosensor it is not. Pharmacol Rev. 61(3): 228-261. doi:10.1124/pr.109.001263. PMID:19749171.

Rukwied, R., Chizh, B. A., Lorenz, U., Obreja, O., Margarit, S., Schley, M., et al. 2007. Potentiation of nociceptive responses to low pH injections in humans by prostaglandin E2. J Pain. 8(5): 443-451. doi: 10.1016/j.jpain.2006.12.004. PMID: 17337250.

Safari, F., Aminnejad, R., Mohajerani, S. A., Farivar, F., Mottaghi, K., \& Safdari, H. 2016. Intrathecal Dexmedetomidine and Fentanyl as Adjuvant to Bupivacaine on Duration of Spinal Block in Addicted Patients. Anesth Pain Med. 6(1): e26714. doi:10.5812/aapm.26714. PMID: 27110524.

Sagie, I., \& Kohane, D. S. 2010. Prolonged sensory-selective nerve blockade. Proc. Natl. Acad. Sci. U.S.A. 107(8): 3740-3745. doi:10.1073/pnas.0911542107. PMID:20133669.

Shankarappa, S. A., Sagie, I., Tsui, J. H., Chiang, H. H., Stefanescu, C., Zurakowski, D., et al. 2012. Duration and local toxicity of sciatic nerve blockade with coinjected site 1 sodium-channel blockers and quaternary lidocaine derivatives. Reg Anesth Pain Med. 37(5): 483-489. doi: 10.1097/AAP.0b013e31826125b3. PMID:22914659.

Thalhammer, J. G., Vladimirova, M., Bershadsky, B., \& Strichartz, G. R. 1995. Neurologic evaluation of the rat during sciatic nerve block with lidocaine. Anesthesiology. 82(4): 1013-1025. PMID:7717536.

Zhou, C., Liang, P., Liu, J., Zhang, W., Liao, D., Chen, Y., et al. 2014. Emulsified isoflurane enhances thermal transient receptor potential vanilloid-1 channel activation-mediated sensory/nociceptive blockade by QX-314. Anesthesiology. 121(2): 280-289. doi:10.1097/ALN.0000000000000236. PMID: 24667830. 


\section{Figure captions}

Fig. 1. Time courses of sciatic nerve block after injection of $35 \mathrm{mM}$ QX-314 with or without three concentrations of dexmedetomidine in rats $\left(n=8 \cdot \operatorname{group}^{-1}\right)$. (A) The PWLs were enhanced when treated with $35 \mathrm{mM}$ QX-314 plus $5.3 \mu \mathrm{M}, 26.4 \mu \mathrm{M}$ and $52.8 \mu \mathrm{M}$ dexmedetomidine. (B) $35 \mathrm{mM}$ QX-314 could not effectively block the motor function of rats' sciatic nerves, but coadministration of QX-314 with dexmedetomidine resulted in effective motor blockade that lasted for 5-8 h. (C-D) From post-drug $15 \mathrm{~min}$ to $10 \mathrm{~h}$, statistically significant differences were found at individual time points of both thermal nociception and motor function blockade between $5.3 \mu \mathrm{M}$ dexmedetomidine/35 mM QX-314 combination and $35 \mathrm{mM}$ QX-314 alone. Data are shown as median and 25th percentile. The dotted lines (PWL $=6 \mathrm{~s}$; Motor score $=2$ ) mean the boundaries between effective and ineffective blockades, above being effective blockades. ${ }^{*} P<0.05$; ${ }^{*} P<0.01 ; * * * P<0.001$. Abbreviations: PWL, paw withdrawal latency; DEXM, dexmedetomidine.

Fig. 2. Duration of effective block between $35 \mathrm{mM}$ QX-314 with or without dexmedetomidine at different concentrations $(5.3 \mu \mathrm{M}, 26.4 \mu \mathrm{M}$ and $52.8 \mu \mathrm{M}, \mathrm{n}=8$ rats $\cdot$ group $^{-1}$ ). Dexmedetomidine added to $35 \mathrm{mM}$ QX-314 significantly prolonged the duration of effective thermal nociception blockade (A) and motor blockade (B) when compared with $35 \mathrm{mM}$ QX-314 alone, but not in a dose-dependent fashion when comparing the groups of three concentrations of dexmedetomidine/QX-314 $(P>$ 0.0083 and detailed $P$ values noted in the figure). The centre line is the median, the lower and upper boundaries are the 25th and 75th percentiles, and the error bars are the minimum and maximum. \# $P<0.05 / 6$ (0.0083). Abbreviation: DEXM, dexmedetomidine. 
Fig. 3. Thermal nociception blockade after injection of dexmedetomidine $(5.3 \mu \mathrm{M}$, $26.4 \mu \mathrm{M}$ and $52.8 \mu \mathrm{M})$ alone or added to $35 \mathrm{mM} \mathrm{QX}-314\left(\mathrm{n}=8\right.$ rats $\cdot$ group $\left.^{-1}\right)$. (A, C, E) Injection of three doses of dexmedetomidine alone did not produce effective thermal nociception blockade. (B, D, F) The analgesic effects of different concentrations of dexmedetomidine added to QX-314 were significantly greater in the operation paws than the control paws. Abbreviations: DEXM, dexmedetomidine; PWL, paw withdrawal latency.

Fig. 4. Results of haematoxylin-eosin staining at day $14\left(n=8\right.$ sections $\left.^{\prime} \operatorname{group}^{-1}\right)$. (A) In the inflammation score, there were no statistical differences between $23 \mathrm{mM}$ bupivacaine (from the preliminary study as a positive control) and $35 \mathrm{mM}$ QX-314 with or without dexmedetomidine $(5.3 \mu \mathrm{M}$ and $26.4 \mu \mathrm{M})(P>0.005)$. But $52.8 \mu \mathrm{M}$ dexmedetomidine added to QX-314 showed less inflammatory response relative to QX-314 alone. (B) None of the samples showed obvious signs of degeneration or regeneration of muscle. $※ P<0.05 / 10(0.005)$.

Fig. 5. Recordings of compound action potentials (CAPs) from frog sciatic nerve fibres $\left(n=6 \cdot\right.$ group $\left.^{-1}\right)$. (A) The relative peak amplitudes of CAPs from sciatic nerve fibres treated with dexmedetomidine at two concentrations for $60 \mathrm{~min}$. Compared with baselines (the average values for 20 min before giving drugs), the relative CAPs amplitudes were not significantly changed by dexmedetomidine $(26.4 \mu \mathrm{M}$ and 52.8 $\mu \mathrm{M})$. (B) Results of relative amplitudes of CAPs of QX-314 (35 mM) with or without dexmedetomidine $(5.3 \mu \mathrm{M}, 26.4 \mu \mathrm{M}$ and $52.8 \mu \mathrm{M})$. Statistical differences were found between both of four groups $(P<0.05)$. Typical CAPs of four groups are presented in 
Figure 5C, showing post-drug 0 min (baselines, not being soaked with test solution), $15 \mathrm{~min}$ and $60 \mathrm{~min}$, respectively. Data are shown as mean $\pm \mathrm{SEM}$. 


\section{A}
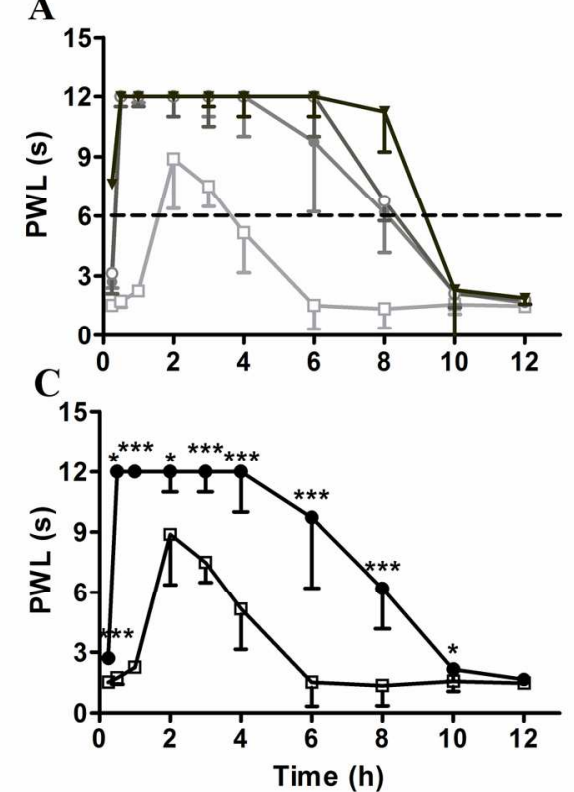
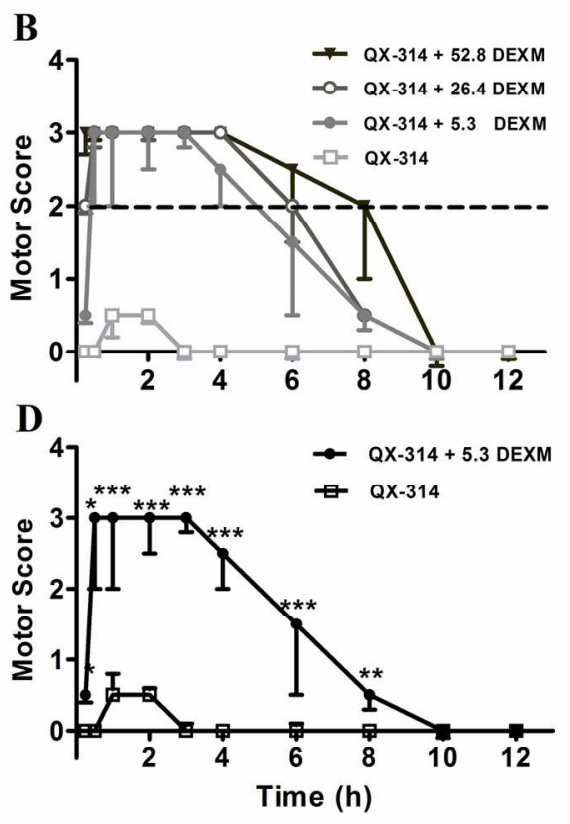

Fig. 1. Time courses of sciatic nerve block after injection of $35 \mathrm{mM} \mathrm{QX}-314$ with or without three concentrations of dexmedetomidine in rats ( $n=8 \bullet$ group -1$)$.

$81 \times 57 \mathrm{~mm}(600 \times 600 \mathrm{DPI})$ 

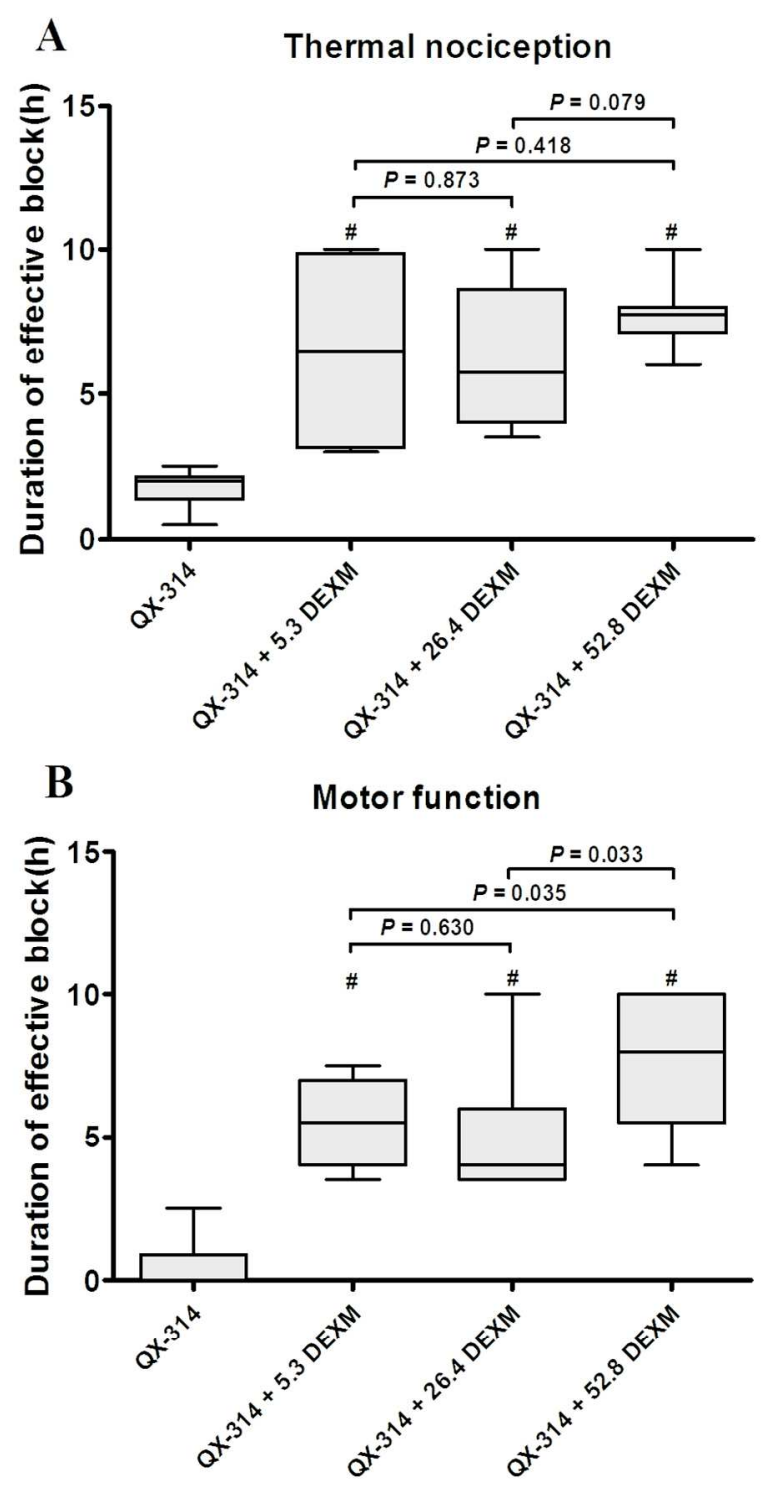

Fig. 2. Duration of effective block between $35 \mathrm{mM}$ QX-314 with or without dexmedetomidine at different concentrations $(5.3 \mu \mathrm{M}, 26.4 \mu \mathrm{M}$ and $52.8 \mu \mathrm{M}, \mathrm{n}=8$ rats•group-1).

$95 \times 163 \mathrm{~mm}(300 \times 300$ DPI $)$ 
A

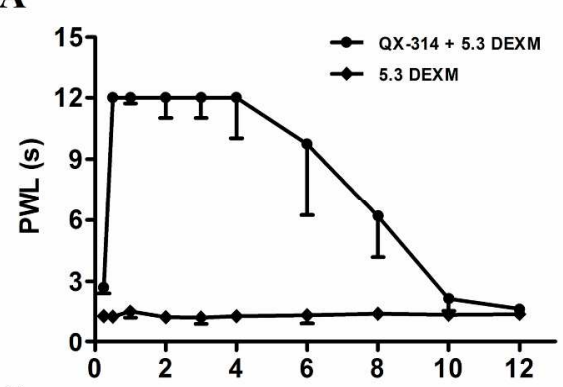

C

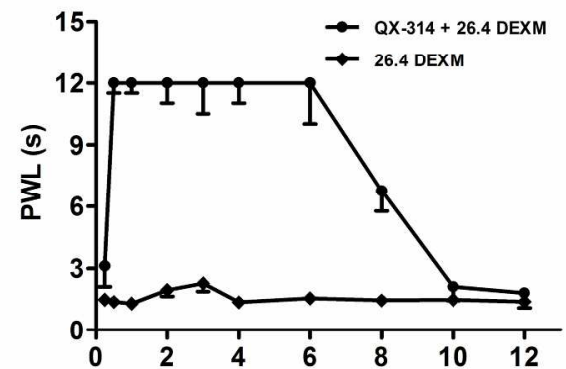

E

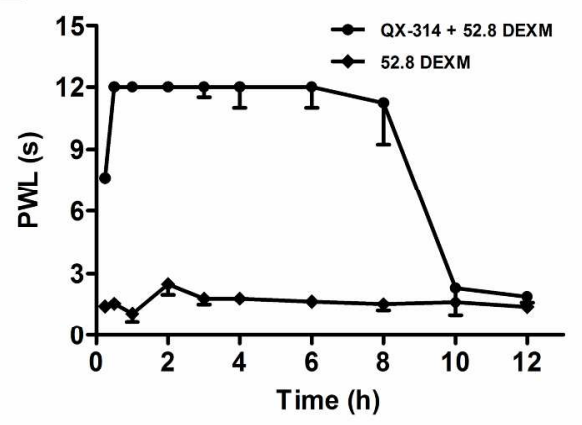

B

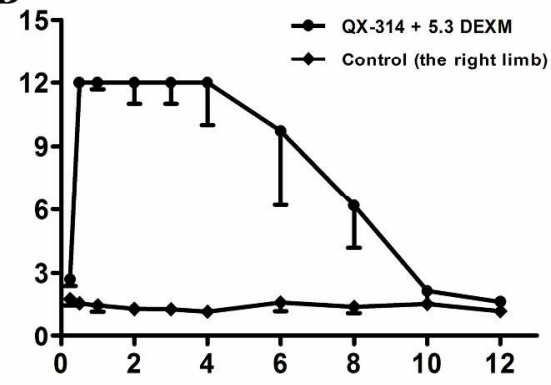

D

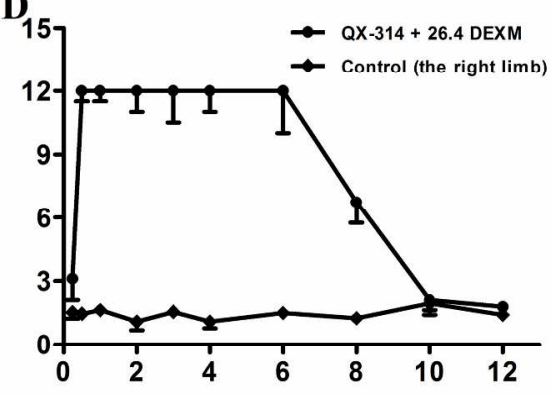

F

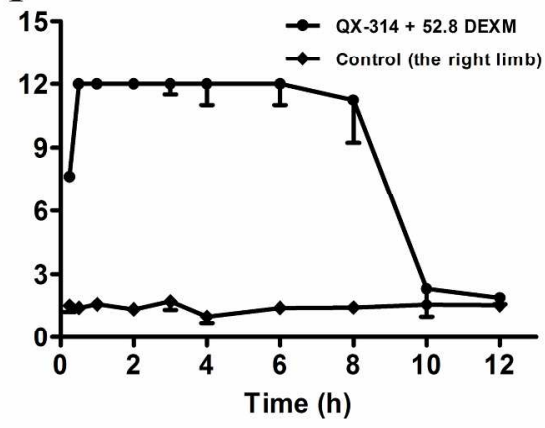

Fig. 3. Thermal nociception blockade after injection of dexmedetomidine $(5.3 \mu \mathrm{M}, 26.4 \mu \mathrm{M}$ and $52.8 \mu \mathrm{M})$ alone or added to $35 \mathrm{mM}$ QX-314 ( $\mathrm{n}=8$ rats・group-1).

$120 \times 126 \mathrm{~mm}(600 \times 600 \mathrm{DPI})$ 

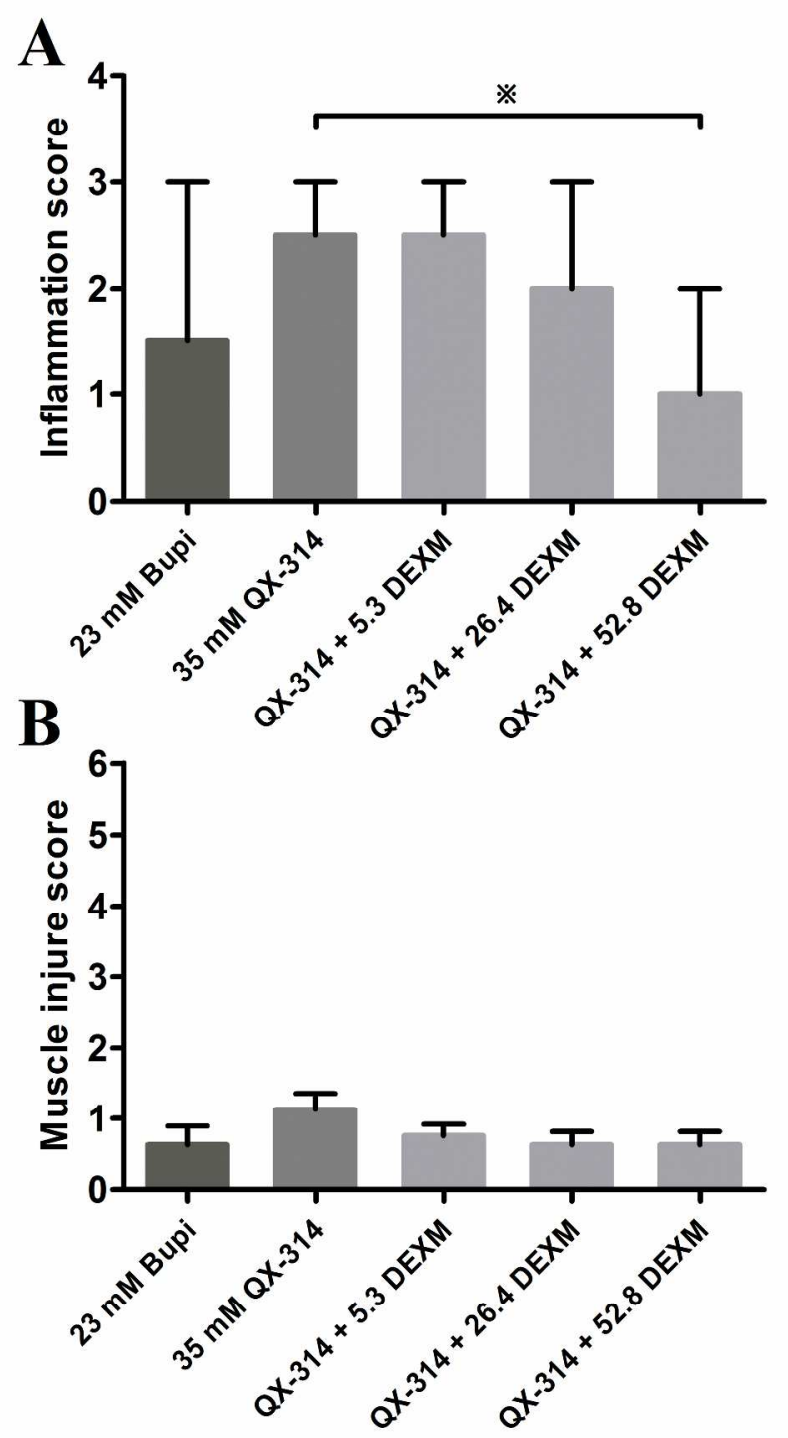

Fig. 4. Results of haematoxylin-eosin staining at day 14 ( $n=8$ sections $\bullet$ group-1). $110 \times 201 \mathrm{~mm}(600 \times 600 \mathrm{DPI})$ 


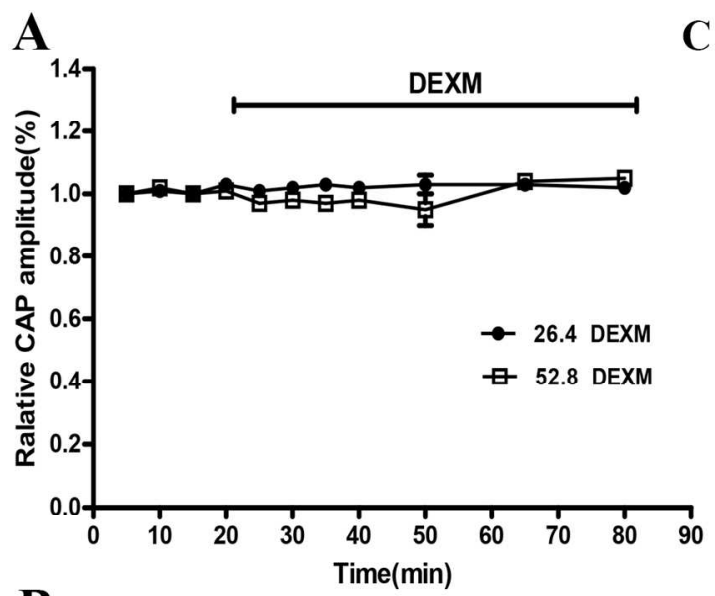

C
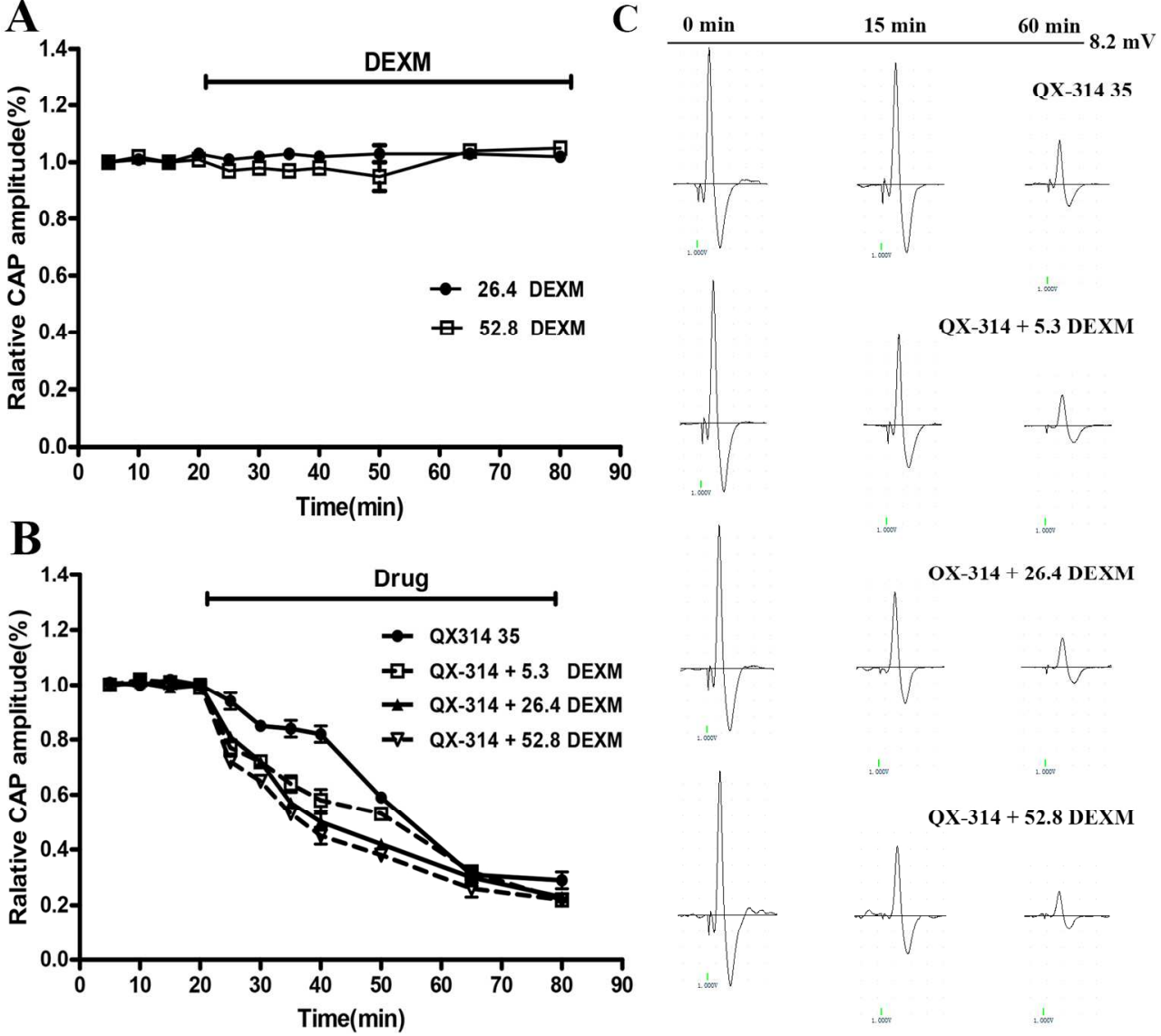

Fig. 5. Recordings of compound action potentials (CAPs) from frog sciatic nerve fibres ( $n=6 \bullet$ group-1).

$152 \times 138 \mathrm{~mm}(300 \times 300 \mathrm{DPI})$ 


\section{Supplemental material -- preliminary experiment}

TABLE 1. Successful sciatic nerve blocks of different agents in preliminary experiment.

\begin{tabular}{lcccc}
\hline \multirow{2}{*}{$\begin{array}{c}\text { Concent } \\
\text { mmol/L }\end{array}$} & $\mathbf{n}$ & \multicolumn{2}{c}{ Number of successful blocks } \\
\cline { 3 - 5 } & $/$ & & Sensory & Motor \\
\hline Saline & 23 & 8 & 0 & 0 \\
Bupivaciane & 25 & 8 & 8 & 8 \\
QX-314 & 35 & 8 & 4 & 1 \\
& 45 & 8 & 8 & 8 \\
\hline
\end{tabular}

Each rat received a $0.2 \mathrm{~mL}$ solution injection on left leg. Sensory block was determined in the same anatomic area using a hotplate at a temperature of $56^{\circ} \mathrm{C}$, successful sensory block was defined as the first time point when thermal latency was greater than $6 \mathrm{~s}$. Motor block was measured as grams exerted in hind paw push-off on an upright balance using a digital platform balance, successful motor block was defined as the value was lower than $50 \%$ of baseline. At day 14 , we harvested the adjacent nerve and muscle tissues of eight rats in group bupivacaine for haematoxylin-eosin staining to test the tissue reaction. 